\title{
Compromission de la masse osseuse et de la motricité chez des malades traités par blocage androgénique
}

\author{
Giandomenico PASSAVANTI, Valerio PIZZUTI, Massimo TOSTI BALDUCCI ${ }^{1}$, Riccardo PAOLINI
}

Service d'Urologie, ${ }^{1}$ Service de Médecine Nucléaire, Hôpital "Misericordia",Grosseto. Italie

\section{RESUME}

Le blocage androgénique thérapeutique détermine un hypogonadisme qui se reflète sur l'appareil locomoteur des malades. Nous avons essayé de vérifier ces effets sur un groupe de malades porteurs d'un cancer de la prostate et présentant une espérance de vie longue.

Trente-six malades soumis à une prostatectomie radicale (PSA moyen : 7,2 $\pm 1,3 \mathrm{ng} / \mathrm{ml}$ ), avaient un stade pT3 dans 24 cas et pT2c dans 12 ; le premier groupe a eu une radiothérapie et un blocage androgénique, et le deuxième un blocage androgénique seul après la chirurgie. Après 2436 mois (moyenne $=28,2$ mois), nous avons réalisé un bilan d'extension avec scanner, scintigraphie osseuse, dosage de PSA et testostérone, et densitomètrie osseuse ; un bilan identique a été répété 53,1 mois en moyenne, après le début du traitement. Le bilan d'extension n'a jamais mis en évidence de reprise de la maladie ; le PSA était compris entre 0,01 et $0,4 \mathrm{ng} / \mathrm{ml}$ (moyenne : $0,11 \pm$ $0,96 \mathrm{ng} / \mathrm{ml}$ ) et la testostérone était de $0,4 \mathrm{ng} / \mathrm{ml}$ en moyenne.

La première densitométrie révélait une ostéopénie : T score $=-1,71 \pm 0,91 ;$ Ward score $=-2,22 \pm 0,917 ; \mathrm{BMD}$ (densité osseuse) $=0,879 \pm 0,126$. La seconde densitométrie montrait une évolution vers l'ostéoporose et une réduction significative de $6 \%$ de la BMD : T Score $=-1,95$ $\pm 0,84$; Ward score $=-2,4 \pm 0,87 ; B M D=0,819 \pm 0,12$.

Pendant cet intervalle de temps, 3 patients ont eu une fracture du fémur et un quatrième une fracture de deux côtes après un effort physique. Tous les patients se plaignaient d'avoir une réduction de la force physique et une très importante fatigabilité.

On peut conclure que le blocage androgénique est la cause d'une altération de la motricité et de la qualité de vie des malades traités pour un cancer de la prostate et bénéficiant d'une grande espérance de vie.

Mots clés : blocage androgénique, densitométrie osseuse, masse osseuse, ostéoporose

\section{INTRODUCTION}

L'hypogonadisme secondaire à un blocage androgénique est la cause de plusieurs effets collatéraux. La testostérone exerce une action trophique sur nombreux organes et tissus, ainsi que sur l'appareil locomoteur [9]. Nous avons essayé de vérifier les effets de cette thérapie sur la masse osseuse [8] et sur la motricité chez un groupe de malades porteurs d'un cancer de prostate présentant une longue espérance de vie.

\section{MATERIELS ET METHODES}

Trente six patients, âgés de 59 à 74 ans (moyenne 69,8 ans), ont été opérés par prostatectomie radicale (PR) pour cancer de prostate. Selon le stade histopathologique les patients étaient repartis de la façon suivante : vingt-quatre patients pT3, dont 14 pT3a et 10 pT3b; 12 patients étaient pT2c.

Les 24 malades pT3 ont été traités par radiothérapie conformationnelle et hormonothérapie après intervention chirurgicale. Les 12 patients pT $2 c$ ont eu juste un blocage androgénique après la chirurgie. Le blocage androgénique consistait en un agoniste $\mathrm{LH}-\mathrm{RH}$ et de l'acétate de cyprotérone.

Avant l'intervention chirurgicale les malades avaient eu : un dosage de PSA (moyenne : 7,2 $\pm 1,3 \mathrm{ng} / \mathrm{ml}$ ), une biopsie systématique, un scanner et une scintigraphie osseuse.

Vingt-quatre à trente-six mois (moyenne : 28,4 mois) après le début de la thérapie, tous les malades ont eu un bilan d'extension avec scanner, scintigraphie osseuse, PSA, testostérone, densitomètrie osseuse et évaluation clinique.

\section{Correspondance:}

Dr Giandomenico PASSAVANTI - 44 Rue Oberdan. 58100 Grosseto. Italie - Tel 0039-0564 411864 -

Email mpeppina@infinito.it 
Quarante-huit à soixante-douze mois (moyenne : 51,3 mois) après le début du blocage androgénique, nous avons effectué un bilan d'extension identique. La densitomètrie osseuse était centrée sur le col fémoral (région à risque élevé de fractures) au moyen d'un appareil Hologique avec une méthodologie à $\mathrm{RX}$ et un double rayon photonique. La densitomètrie osseuse à double rayon photonique permet d'examiner une ou plusieurs régions osseuses avec une importante réduction des temps d'exposition aux radiations. Elle mesure le contenu minéral osseux (BMC : Bone Mineral Content) du segment examiné ; le BMC est divisé par le volume osseux examiné pour obtenir la densité minérale osseuse (BMD : Bone Mineral Density) en $\mathrm{gr} / \mathrm{cm} 3$.

Les paramètres suivants ont été évalués : densité osseuse (BMD), T score et Ward score. Le T score est la "Standard deviation" des données relatives aux sujet examiné, en rapport avec les valeurs moyennes calculées sur une population de jeunes adultes (valeur normale : >-1,5). Le score sur le triangle de Ward (valeur normale : $>-1,5$ ) est la même donnée mesurée sur un point, à très haut risque de fractures, du col fémoral, délimité par cinq faisceaux de trabécules osseux, orientés selon la ligne de force.

\section{RESULTATS}

Les deux bilans d'extensions ne démontraient jamais une reprise de maladie.

Le PSA était compris entre 0,01 et $0,4 \mathrm{ng} / \mathrm{ml}$ (moyenne : $0,11 \pm 0,96 \mathrm{ng} / \mathrm{ml}$ ), mais seule 2 sujets avait une valeur de $0,4 \mathrm{ng} / \mathrm{ml}$. Aucun malade n'a pas présenté de lésions néoplasiques secondaires sur la scintigraphie osseuse ou au scanner. La testostérone était comprise entre 0,06 et 0,2 $\mathrm{ng} / \mathrm{ml}$ (moyenne : 0,4 $\mathrm{ng} / \mathrm{ml}$ ).

La première densitométrie faisait apparaître une ostéopénie (Figure 1): T score $=-1,71 \pm 0,91 ;$ Ward score $=-2,22 \pm$ 0,$917 ; \mathrm{BMD}=0,879 \pm 0,126$.

La deuxième densitométrie montrait une réduction statistiquement significative (Wilcoxon test : $Z=5,22$ ), de $6 \%$ de la densité osseuse, ainsi qu'une évolution vers l'ostéoporose : T score $=-1,95 \pm 0,84 ;$ Ward score $=-2,4 \pm 0,87 ; B M D=$ $0,819 \pm 0,12$.

Pendant cet intervalle de temps, trois patients ont eu une fracture d'allure ostéoporotique du fémur et un patient une fracture de deux côtes après un effort physique. Tous les patients se plaignaient d'avoir une réduction de la force physique et une excessive fatigabilité avec une dégradation de la motricité.

\section{DISCUSSION}

Le blocage androgénique est employé depuis longtemps dans la thérapie du cancer de la prostate [16]. Pendant les 20 dernières années, de nombreuses méthodologies diagnostiques et thérapeutiques ont été introduites dans l'usage clinique. Celles-ci ont permis d'obtenir des longues survies et donc de longues périodes de thérapie hormonale.
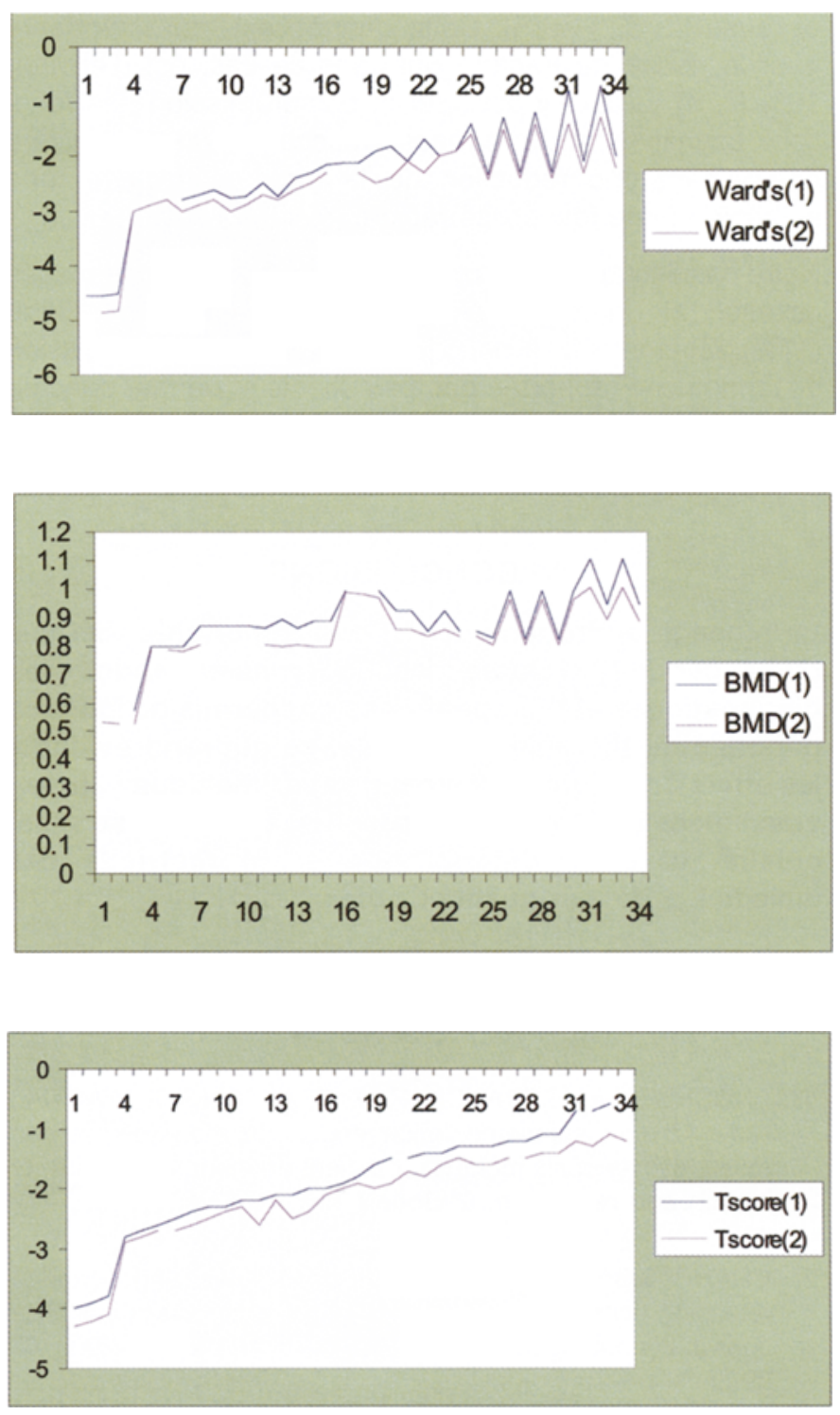

Figure 1 : Distribution graphique du Ward score, $T$ score et de la densité osseuse (BMD) évalués 24-36 mois (1) et 48-72 mois (2) après le début de la déprivation androgénique ; on peut apercevoir une persistante évolution vers l'ostéoporose.

L'hypogonadisme, induit par cette thérapie, est responsable de nombreuses modifications métaboliques qui peuvent altérer la qualité de vie des malades. II est bien connu que la testostérone exerce une action trophique sur la masse osseuse soit pendant la croissance [13], soit à l'âge adulte $[1,8,12]$. Cette action, sur les ostéoblastes [5], est régulée par les facteurs de croissance [4] ; elle est déterminée par la testostérone, soit directement, soit après transformation en oestradiol [1]. La testostérone, enfin, favorise la synthèse du 1-25 dihydroxycholécalciférol [14] et la sécrétion de calcitonine [4]. La testostérone a une action trophique sur la masse musculaire et réduit la masse grasse [7] ; elle améliore les fonctions cognitives [10].

Le blocage androgénique détermine ainsi, lentement et progressivement [6], un appauvrissement de la masse 
osseuse $[2,3,11,15]$. Cette donnée est très importante pour le risque de fracture qui dans ce cas, est d'environ $10 \%[3,4]$; il faut y associer la compromission des fonctions cognitives et de la masse musculaire, cette dernière déterminant une réduction de la force musculaire, une asthénie et une fatigabilité rapide.

Notre casuistique révèle une perte significative de la masse osseuse et un risque élevé de fracture ostéoporotique $(11 \%)$. Ceci vérifie la notion subjective de compromission de la motricité rapportée par les patients et permet donc de montrer que cette condition peut compromettre la qualité de vie de malades traités par blocage androgénique.

\section{CONCLUSIONS}

Le blocage androgénique est une importante thérapie du cancer de la prostate; les modernes méthodologies diagnostiques et thérapeutiques ont permis de longues périodes de thérapie hormonale, ce qui rend évidents les effets collatéraux du blocage androgénique : appauvrissement de la masse osseuse et de la masse musculaire, et enfin détérioration de la motricité des malades et de leur qualité de vie.

\section{REFERENCES}

1. ANDERSON F.H., FRANCIO R.M., PEASTON R.T., WASTELL H.J. : Adrogen supplementation eugonadal men with osteoporosis : effects of six months' treatment on markers of bone formation and resorbtion. J. Bone Miner. Res., 1997, 12 : 472478.

2. CHANG S.S. : Exploring the effects of luteinizing hormonereleasing hormone agonist therapy on bone health : implications in the management of prostate cancer. Urology, 2003, 62: 29-35.

3. DANIEL H.W. : Osteoporosis after orchiectomy for prostate cancer. J. Urol., 1997, $57:$ 439-444.

4. DAHMANI L., WAGNER B., AUZANNEAU C., IRANI J., DORE $B$. : Prévalence des fractures d'allure ostéoporotique chez des patients traités par blocage androgénique pour cancer de prostate. Prog. Urol., 2003, $13:$ 73-78.

5. GOLDRAY D., WEISMAN Y., JACCARD N., MERDLER C., CHEN J., MATZKIN H. : Decreased bone density in elderly men treated with the gonadotropin-realesing hormone agonist D-Trp6-GnRh. J.Clin. Endocrin. Metab., 1993, 76 : 288-290.

6. HUSSAIN S.A., WESTON R., STEPHENSON R.N., GEORGE E., PARR N.J. : Immediate dual energy Xray absorptiometry reveals a high incidence of osteoporosis in patients with advanced prostate cancer before hormonal manipulation. Br. J. Urol. Int., 2003, 92 : 690-694.

7. KUHN J.M. : Déficit androgénique : effet de la substituition sur la composition corporelle. Andrologie, 2002, $12: 17-23$.

8. LEGRAND E., SIMON V., AUDRAN M. : Stéroides sexuels et ostéoporose chez l'homme. Andrologie, 2002,12 : 24-31.

9. LEJEUNE H. : Le déficit androgénique lié à l'âge chez l'homme : terminologie, épidémiologie. Andrologie, 2002, 12 : 11-16.

10. MIMOUN S. : Androgènes et qualité de vie. Andrologie, 2002, $12: 257-261$.
11. MITTAN D., LEE S., MILLER E., PEREZ R.C., BASLER J.W., BRUDER J.M. : Bone loss following hypogonadism in men with prostate cancer treated with $\mathrm{GnRH}$ analogs. J. Clin. Endocrin. Metab., 2002, 87 : 3656-3661.

12. MURPHY S., KHAW K.T., CASSIDY A., COMPSTON J.E. : Sex hormones and bone mineral density in elderly men. Bone Miner., 1993, $20: 133-140$.

13. NUTI R., GENNARI C. : L'osteoporosi maschile. X Congr. Naz., S.I.A., $1996: 577-585$.

14. OTREMSKI I., LEV-RAN M., SALAMA R., EDELSTEIN S. : The metabolism of vitamine D3 in response to testosterone. Calcif. Tissue Int., 1997, $60: 485-487$.

15. PASSAVANTI G., PIZZUTI V., BRAGAGLIA M., TOSTI BALDUCCI M., PAOLINI R. : Hormone therapy induced hypogonadism on prostatic Ca-patients : can it actually significantly alter bone metabolism. Giorn. It. Androl., 1998, $5: 51-53$.

16. SCHRODER F. : Terapia ormonale del carcinoma prostatico. In: Urologia di Campbell. sett.ediz.ital. Verduci, 1999, 5 : 26612678.

Manuscrit reçu : juin 2004 ; accepté août 2004.

Reduction of bone mass and motility in patients treated with androgen deprivation therapy

\section{Giandomenico PASSAVANTI, Valerio PIZZUTI, Massimo TOSTI BALDUCCI, Riccardo PAOLINI}

Therapeutic androgen suppression induces hypogonadism with effects on the patient's locomotor system. We tried to verify these effects on a group of patients with prostate cancer presenting a prolonged life expectancy.

Thirty six patients treated by radical prostatectomy (mean PSA: $7.2 \pm 1.3 \mathrm{ng} / \mathrm{ml}$ ) had stage pT3 cancer in 24 cases and pT2c in 12 cases.

The first group was treated by radiotherapy and androgen suppression and the second group was treated by androgen suppression alone after surgery. After 24-36 months (mean $\mathbf{2} \mathbf{2 8 . 4}$ months), staging was performed by CT scan, bone scintigraphy, PSA and testosterone assays, and bone densitometry. An identical assessment was repeated an average of $\mathbf{5 3 . 1}$ months after starting treatment. Staging never demonstrated disease recurrence; PSA was between 0.01 and $0.4 \mathrm{ng} / \mathrm{ml}$ (mean: $0.11 \pm 0.96 \mathrm{ng} / \mathrm{ml}$ ) and the mean plasma testosterone was $0.4 \mathrm{ng} / \mathrm{ml}$.

The first bone densitometry revealed osteopenia: T score $=-1.71 \pm 0.91$; Ward score $=-2.22 \pm 0.917$; BMD (bone density) $=0.879 \pm 0.126$. The second bone densitometry showed progression to osteoporosis and a significant $6 \%$ 
reduction of the BMD: $\mathrm{T}$ Score $=-1.95 \pm 0.84$; Ward score $=$ $-2.4 \pm 0.87 ; B M D=0.819 \pm 0.12$.

During this time interval, 3 patients developed a fracture of the femur and a fourth patient fractured two ribs after physical exertion. All patients complained of decreased physical strength and very marked fatigability.

We can conclude that androgen suppression causes an alteration of locomotor function and quality of life of patients treated for prostate cancer and presenting a long life expectancy.

Key Words : androgen suppression, bone densitometry; bone mass, osteoporosis 